\title{
Bone Trabecular Analysis of Femur Radiographs for the Assessment of Osteoporosis Using DWT and DXA
}

\author{
V. Sapthagirivasan, M. Anburajan, and V. Mahadevan, Member, IACSIT
}

\begin{abstract}
The lifespan can be increased in a positive mode by initial diagnosis of osteoporosis. Analysis of trabecular properties on digital hip radiographs could be useful in identifying the subjects with low bone mineral density (BMD) or osteoporosis. Early detection of fracture risk is important for initiating treatment and improving outcomes from both physiologic and pathologic causes of bone loss. So, the aim of the present study was to test the potentiality of the discrete wavelet transform based qualitative trabecular bone analysis method for the assessment of osteoporosis using simple radiographs. Our study was performed on 20 post-menopausal women without osteoporotic fractures and 21 healthy pre-menopausal women. For all subjects radiographs and bone mineral density measurements were obtained. The energy computed from trabecular pattern of normal bone by trabecular enhanced radiographic samples appeared to be higher than the energy from samples of the osteopenia and osteoporosis in greater-trochanter and neck regions. Hence forth, our results suggest that, the plain radiographs might be used instead of BMD measurements for the screening of osteoporosis and its associated fracture risk assessment.
\end{abstract}

Index Terms-Osteoporosis, osteopenia, digital radiograph, texture analysis, discrete wavelet transform (DWT), BMD, DXA, Indian women.

\section{INTRODUCTION}

With the better of life expectancy, the risk of facing diseases caused by aging process is increasing. One of those diseases is the loss of bone mass or osteoporosis. Osteoporosis is a worldwide medical abnormality affecting middle-aged and older populations, especially women. In INDIA, one in three women and one in eight men over the age of 50 will suffer, a fracture attributed to osteoporosis [1]. Fractures happen at a great personal and socioeconomic cost. Twenty percent of people who have a hip fracture die within 12 months, half of those who survive can no longer live independently, almost half of those who could walk unaided are no longer able to do so and a quarter are still unable to prepare their own dinner [2]-[4]. Earlier detection of osteoporosis can be done by using Bone Mineral Densitometry (BMD) technique using various modes such as ultrasound or Dual Energy X-ray Absorptiometry (DXA). For the time being, DXA is considered as a gold standard for

Manuscript received November 20, 2012; revised January 23, 2013. This work was presented at the International Conference (ICSCT) Kunming, China during 2010.

V. Sapthagirivasan and M. Anburajan are with the SRM University, Department of Biomedical Engineering, Kattankulathur, Chennai, India 603203 (e-mail: sapthagiri.ece@gmail.com, anbufelix@yahoo.com).

V. Mahadevan is with the Swinburne University of Technology, Department of Information Systems and e-Business, Melbourne, Australia (e-mail: vmahadevan@swinburne.edu.au). detection of Osteoporosis. Osteoporosis is defined by the World Health Organization as a BMD lower by more than 2.5 standard deviations than the mean value for young adults. As people grow older, particularly Postmenopausal women, they lose bone mass and become more susceptible to osteoporosis and fracture [5]. BMD alone cannot accurately predict the fracture, as other factors such as the shape and structure of bone and the risk of falling come into picture. The architecture of the bone is composed by the cortical bone shell and trabecular bone core. The Trabecular bone is a spongy, porous type, found at the ends of all bones, such as pelvis and spine [6]. In proximal femur, trabecular bone forms a pattern of net-like strands varying in thickness and numbers [7] as shown in Fig. 1. It has a complex three dimensional structure consisting of struts and plates.

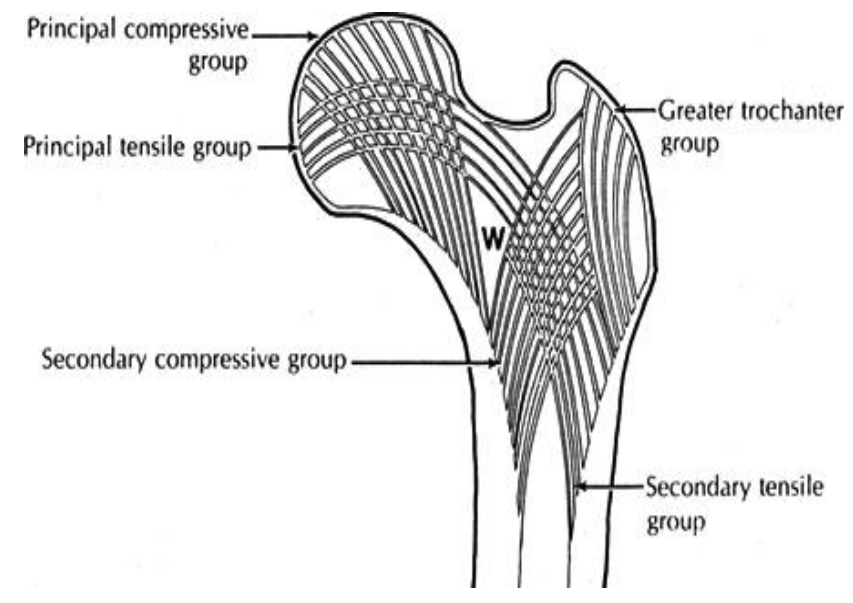

Fig. 1. Trabecular structure of the femur bone

Many lines of evidence indicate that the decreased bone strength which is the characteristic of osteoporosis is dependent not only on BMD, but also on trabecular bone micro-architecture and mineralization. The correlation between bone strength and bone mass is well established but the relationship between trabecular micro-architecture and bone mass are less explored.

Trabecular patterns appearing on digital X-rays contain rich information about the bone status. The observation of trabecular pattern change for diagnosis of osteoporosis was first proposed in the 1960s using radiographs of proximal femur. The diagnosis was known as Singh Index visual grading system [3]. A number of physicians, due to the lack of diagnosis equipment like DXA, observe the trabecular change visualize in proximal femur recorded in radiographs to assess osteoporosis [5], [6]. On radiographs, femur (trabecular) bone structure appears as a distinct pattern.

To solve the variability problem of Singh index grading system, we proposed the texture analysis system for osteoporosis assessment by observing trabecular change in 
proximal femur. Gabor filter, wavelet transforms and fractal analysis algorithms will be applied to extract the features of trabecular pattern recorded on proximal femur radiographs. Initial research in features extraction of proximal femur trabecular pattern using Gabor filter and discrete wavelet transform (DWT) has been performed with quite promising result [7]-[9]. The extracted features will represent the quality or structure of the bone, better quality represents better bone strength, lower quality leads to low bone strength and could be suspected as osteoporotic. The extracted features of the samples, in the form of energy, are then compared with their corresponding BMD obtained by DXA.

The aim of the present study was to test the potentiality of the Gabor wavelet transform method in the evaluation of osteoporosis by comparing with the central DXA as a gold standard.

\section{MATERIALS AND METHOD}

\section{A. Subjects and Procedures}

The present study emphasizes the novel ideas with respect to osteoporotic abnormality within the framework of public health. . A free screening camp for osteoporosis diagnosis was organized by the end of the year 2010 at SRM University. All the participants had signed informed consent form and the study protocol had been cleared by institutional ethical committee. This study was performed on 20 healthy pre-menopausal women $(n=20,34.67 \pm 6.8$ years $)$ and 21 post-menopausal women $(n=21,55.77 \pm 14.9$ years $)$ with no previous diagnosis of trauma or trivial fractures. Bone mineral density (BMD) in $\left(\mathrm{g} / \mathrm{cm}^{2}\right)$ from the femoral neck (FN-BMD), femoral shaft (FS-BMD), femoral wards (FW-BMD), femoral trochanter (FTr-BMD) and total Femur (FT-BMD) were measured by dual-energy X-ray absorptiometry (GE Healthcare Lunar DPX). The study adopted WHO diagnostic criteria to sort osteoporosis. The studied population was divided in to the following groups: Normal (T-score $\geq-1)$; Osteopenia $(-2.5<\mathrm{T}$-score $<-1)$; and Osteoporosis (T-score <-2.5) on the basis of FT-BMD values. For all the subjects, the radiograph of the right femur was obtained with a digital X-ray machine (Multiphos, Siemens, Germany) at 45 to $80 \mathrm{kV}, 2 \mathrm{~mA}$. The femur region would be carefully rotated internally by $15^{\circ}$, while the images are acquired.

\section{B. Bone Trabecular Feature Extraction}

Typically, there is wide variation in the intensity of digital $\mathrm{X}$-ray image from different patients. Hence, it is necessary to identify a reference frame and normalize the intensities of all other images against it. We performed this intensity normalization step using histogram specification [10]. This modifies the image values through a histogram transformation operator which maps a given intensity distribution $a(x, y)$ into a desired distribution $c(x, y)$ using a histogram equalized image $b(x, y)$ as an intermediate stage. Then, this process is applied independently to each individual sub-blocks (window) of $3 \times 3$.

After normalization, algorithm detailed by Sinthanayothin et al [11] for contrast enhancement was used to accelerate both the bone contrast attribute as well as total image intensity. The aim is to apply, a transformation of the values inside small windows in the image in a way that all values are distributed around the mean and shows all possible intensities.

Once the image has been pre-processed as described above, we performed the Gabor and wavelet (4 level decomposition) transform operation to the classification of osteoporosis based on the change of trabecular pattern. The classification was based on features extracted by Gabor and wavelet in the form of energy based on the following equation.

$$
E(I)=\frac{1}{M X N} \sum_{i=1}^{M} \sum_{j=1}^{N}|I(m, n)|^{2}
$$

For image $I(m, n)$ with $1 \leq m \leq M$ and $1 \leq n \leq N$

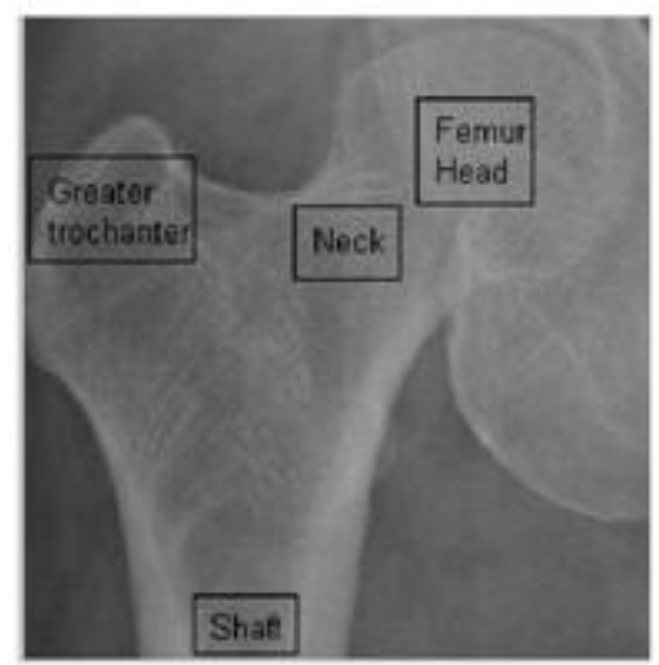

Fig. 2. Right femur digital x-ray image with marked ROIs

Gabor and wavelet features were extracted from various regions of interests (ROIs) of proximal femur such as femoral neck, femoral head, shaft and greater trochanter. Fig. 2 shows the ROIs used in this paper.

\section{RESUlTS AND DISCUSSION}

In this paper, we used four different trabecular pattern recorded in 41 patient's radiograph. The feature is extracted from wavelet features by energy computation and is then compared to trabecular energy computation predetermined trabecular energy.

TABLE I: APPROXIMATION COEFFICIENT VALUES UP TO 4 LEVELS

\begin{tabular}{|l|l|l|c|c|}
\hline \multirow{2}{*}{ Femur regions } & \multicolumn{4}{|c|}{ Energy (gray levels normalized to 0 - 1 scale) } \\
\cline { 2 - 5 } & $\mathbf{A 1}$ & $\mathbf{A 2}$ & $\mathbf{A 3}$ & $\mathbf{A 4}$ \\
\hline Greater Trochanter & 0.496 & 0.621 & 0.589 & 0.557 \\
\hline Lesser Trochanter & 0.643 & 0.647 & 0.554 & 0.549 \\
\hline Neck & 0.657 & 0.665 & 0.584 & 0.583 \\
\hline Head & 0.651 & 0.670 & 0.595 & 0.534 \\
\hline
\end{tabular}

The results obtained by the proposed method were detailed as follows: Fig. 3 details the plotting of energy versus approximation co-efficient with respect to normal, osteopenia and osteoporosis category groups. Fig. 4 enumerates the implementation results of marked ROIs of original and trabecular enhanced. The Table I deciphers the following facts and figures: Greater and lesser trochanter 
region, neck and head region as well, exhibited the highest value at $2^{\text {nd }}$ approximation level. The Table II shows the classification of 3 groups on the basis of the DXA acquired total femur BMD; however, the $4^{\text {th }}$ group has been categorized on chronological basis (less than 30 years of age).
Femoral neck exhibited peak value in both $1^{\text {st }}$ and $4^{\text {th }}$ approximation levels as detailed in Table III. The Table IV depicts the information regarding energy calculation by Gabor wavelet for trabecular enhanced ROIs for all the 3 (Normal, osteopenia and osteoporosis) groups.

TABLE II: BMD OF RIGHT FEMUR ACQUIRED BY DXA

\begin{tabular}{|c|c|c|c|c|c|c|c|}
\hline \multirow{2}{*}{$\begin{array}{c}\text { Group } \\
(n, \text { age } \pm \text { SD years })\end{array}$} & \multirow{2}{*}{$\begin{array}{c}\text { BMI } \\
\left(\mathrm{kg} / \mathbf{m}^{2}\right)\end{array}$} & \multicolumn{5}{|c|}{ Proximal Femur BMD by DXA $\left(\mathrm{g} / \mathrm{cm}^{2}\right)$} & \multirow{2}{*}{$\begin{array}{c}\text { T-score } \\
\text { (SD) }\end{array}$} \\
\hline & & Trochanter & Neck & Ward & Shaft & Total & \\
\hline $\begin{array}{l}\text { Normal } \\
(n=8,55 \pm 3.1)\end{array}$ & $27.5 \pm 4.5$ & $0.76 \pm 0.09$ & $0.91 \pm 0.11$ & $0.73 \pm 0.1$ & $1.19 \pm 0.08$ & $0.98 \pm 0.08$ & $-0.21 \pm 0.63$ \\
\hline $\begin{array}{l}\text { Osteopenia } \\
(n=8,46 \pm 15.7)\end{array}$ & $21.4 \pm 2.7$ & $0.61 \pm 0.07$ & $0.76 \pm 0.03$ & $0.61 \pm 0.08$ & $0.81 \pm 0.36$ & $0.81 \pm 0.05$ & $-1.58 \pm 0.38$ \\
\hline $\begin{array}{l}\text { Osteoporosis } \\
(n=5,72.2 \pm 11.9)\end{array}$ & $21.9 \pm 2.6$ & $0.39 \pm 0.11$ & $0.59 \pm 0.08$ & $0.37 \pm 0.09$ & $0.64 \pm 0.17$ & $0.53 \pm 0.12$ & $-3.8 \pm 0.94$ \\
\hline $\begin{array}{l}\text { Young Adult } \\
(n=20,34.67 \pm 6.8)\end{array}$ & $24.26 \pm 3.7$ & $0.83 \pm 0.12$ & $1.02 \pm 0.17$ & $0.87 \pm 0.21$ & $1.23 \pm 0.18$ & $1.05 \pm 0.16$ & $0.34 \pm 1.25$ \\
\hline
\end{tabular}

TABLE III: ENERGY CALCULATED BY GABOR WAVELET FOR ORIGINAL ROIS

\begin{tabular}{|c|c|c|c|c|c|c|c|c|}
\hline \multirow[b]{3}{*}{ Group } & \multicolumn{8}{|c|}{ Proximal Femur Energy by Wavelet for original images (gray levels normalized to 0 -1 scale) } \\
\hline & \multicolumn{2}{|c|}{ Greater Trochanter } & \multicolumn{2}{|c|}{ Lesser Trochanter } & \multicolumn{2}{|c|}{ Femoral Neck } & \multicolumn{2}{|c|}{ Femoral Head } \\
\hline & $\mathbf{A 1}$ & A4 & A1 & A4 & A1 & A4 & A1 & A4 \\
\hline Normal & $0.51 \pm 0.06$ & $0.56 \pm 0.07$ & $0.64 \pm 0.01$ & $0.55 \pm 0.12$ & $0.66 \pm 0.28$ & $0.58 \pm 0.17$ & $0.65 \pm 0.18$ & $0.53 \pm 0.21$ \\
\hline Osteopenia & $0.54 \pm 0.17$ & $0.58 \pm 0.03$ & $0.51 \pm 0.02$ & $0.58 \pm 0.17$ & $0.6 \pm 0.02$ & $0.56 \pm 0.12$ & $0.68 \pm 0.16$ & $0.56 \pm 0.15$ \\
\hline Osteoporosis & $0.66 \pm 0.11$ & $0.57 \pm 0.01$ & $0.61 \pm 0.16$ & $0.54 \pm 0.21$ & $0.64 \pm 0.09$ & $0.58 \pm 0.11$ & $0.68 \pm 0.17$ & $0.54 \pm 0.13$ \\
\hline
\end{tabular}

TABLE IV: ENERGY CALCULATED BY GABOR WAVELET FOR TRABECULAR ENHANCED ROIS

\begin{tabular}{|c|c|c|c|c|c|c|c|c|}
\hline \multirow[b]{3}{*}{ Group } & \multicolumn{8}{|c|}{ Proximal Femur Energy by Wavelet for trabecular enhanced images (gray levels normalized to 0 - 1 scale) } \\
\hline & \multicolumn{2}{|c|}{ Greater Trochanter } & \multicolumn{2}{|c|}{ Lesser Trochanter } & \multicolumn{2}{|c|}{ Femoral Neck } & \multicolumn{2}{|c|}{ Femoral Head } \\
\hline & A1 & A4 & A1 & A4 & A1 & A4 & A1 & A4 \\
\hline Normal & $0.38 \pm 0.06$ & $0.89 \pm 0.17$ & $0.41 \pm 0.01$ & $0.82 \pm 0.2$ & $0.39 \pm 0.22$ & $0.89 \pm 0.17$ & $0.36 \pm 0.21$ & $0.69 \pm 0.21$ \\
\hline Osteopenia & $0.36 \pm 0.14$ & $0.86 \pm 0.13$ & $0.42 \pm 0.06$ & $0.57 \pm 0.17$ & $0.36 \pm 0.05$ & $0.86 \pm 0.21$ & $0.28 \pm 0.16$ & $0.91 \pm 0.15$ \\
\hline Osteoporosis & $0.33 \pm 0.18$ & $0.84 \pm 0.09$ & $0.41 \pm 0.19$ & $0.87 \pm 0.21$ & $0.32 \pm 0.12$ & $0.83 \pm 0.11$ & $0.37 \pm 0.17$ & $0.83 \pm 0.14$ \\
\hline
\end{tabular}

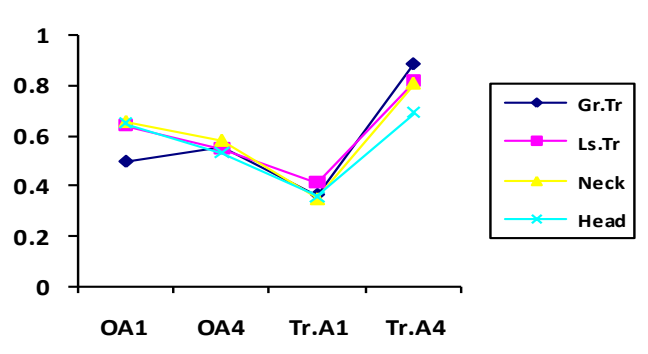

(a)

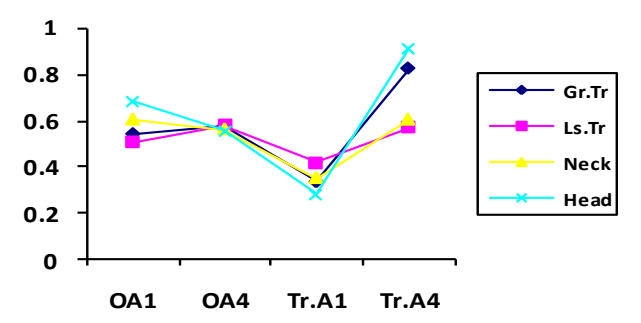

(b)

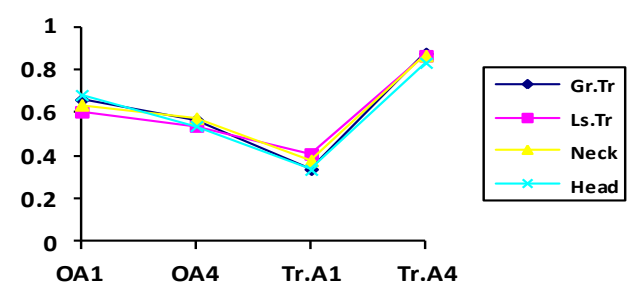

(c)

Fig. 3. Plotting of energy Vs approximation co.eff, (a) Normal, (b) Osteopenia, (c) Osteoporosis

The result of features extraction and energy computation by applying DWT in four scales or levels of decomposition using Gabor wavelet for one radiograph sample is shown in Table I and we found that the significant energies were obtained from level $1^{\text {st }}$ and $4^{\text {th }}$ level decomposition of approximation coefficients. Therefore for the rest of the radiographic samples, the features extraction will be computed for approximation coefficient at $1^{\text {st }}$ and $4^{\text {th }}$ level of decomposition. The energy computed from trabecular pattern of normal bone by trabecular enhanced radiographic samples 
appeared to be higher than the energy from samples of the osteopenia and osteoporosis in greater trochanter and neck regions, whereas the plain radiographs were not showing any significant differences at any sites. The capability of wavelet features is judged in order to assess the degree or level of osteoporosis. The similar study on Gabor wavelet was reported by Pramudito et al [7] in which the feature extracted in the form of energy fractal analysis being utilized by means of box-counting was compared with Singh index scoring and exhibited the positive relativity with bone quality. In yet another similar study Chappard et al [12], detailed about the gradient based texture analysis on hip radiograph from which the fact of close correlation in site-matched areas was figured out in between extracted texture features and BMD.

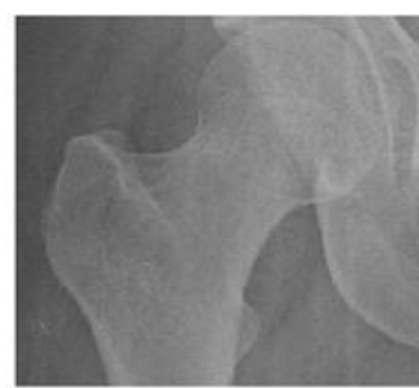

(a)

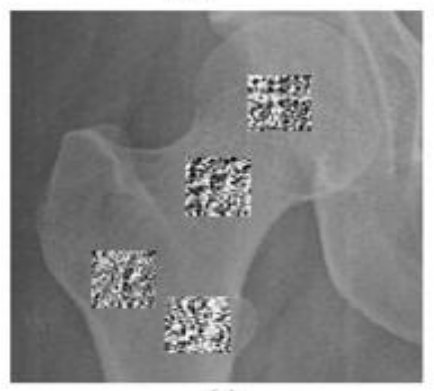

(c)

Fig. 4. Implementation results (a) Original femur x-ray image, (b) Marked region of interests, (c) Trabecular enhanced ROIs, (d) Original ROI of femur head, (e) Enhanced trabecular ROI of (d)

The structural analysis of trabecular bone from clinical radiographs utilizing Radon transform method, gray-level Fourier transform and fractal analysis of heel X-ray images and extracted features in order to discriminate fracture group from the controls as reported by Boehm et al [13] and Chappard et al [14]. However, the finding from this study shows that the healthiest bones, which are having the highest energy and the porous bones, which are having the lowest energy.

\section{CONCLUSION}

Gabor filter and discrete wavelet transforms have been successfully applied to texture analysis of the trabecular pattern recorded in the radiograph of proximal femur. The extracted features from trabecular pattern in the form of energy at greater-trochanter and neck regions were able to give information about the quality of the bones for the assessment of osteoporosis. Hence forth, our results suggest that plain radiographs might be used instead of BMD measurements for the screening of osteoporosis.

\section{APPENDIX}

\section{A. Abbreviations}

BMD: Bone Mineral Density

DXA: Dual Energy X-ray Absorptiometry

BMI: Body Mass Index

DWT: Discrete Wavelet Transform

A1-A4: Approximation co-efficient of DWT at 1 to 4 levels

OA1: Approximation co-efficient of DWT at level 1 from original ROI

OA1: Approximation co-efficient of DWT at level 4 from original ROI

Tr. A1: Approximation co-efficient of DWT at level 1 from trabecular enhanced ROI

Tr. A1: Approximation co-efficient of DWT at level 4 from trabecular enhanced ROI

Gr. Tr: Greater trochanter

Lr. Tr: Lesser trochanter

\section{B. Conflict of Interests}

All the authors declare no conflict of interests related to this manuscript.

\section{Author's Contributions}

All authors contributed equally to the work.

\section{Funding}

All costs and related expenditures have been managed by the main corresponding author only.

\section{ACKNOWLEDGMENT}

The authors wish to thank Department of Orthopedic and Traumatology, Faculty of Medicine, SRM University, for suggesting radiographic guidance and also thankful to Mr. Mariadas and Mr. Dulip, Aarthi Scans, Chennai, India for providing financial concession and their continuous support. They wish to extend their warm regards to $\mathrm{Mr}$. Kishore Mohan KB, Research Scholar, Department of Biomedical Engineering, SRM University for their encouragement and support for organizing camp.

\section{REFERENCES}

[1] Osteoporosis Society of India. Action Plan Osteoporosis: Consensus Statement of an Expert Group, New Delhi, 2003.

[2] M. K. Tony, F. M. Elise, L. N. Glen, and C. Y. Oscar, "Biomechanics of trabecular bone," Annual Review of Biomedical Engineering, vol. 3, pp. $307-333,2001$

[3] P. P. Smyth, J. E. Adams, R. W. Whitehouse, and C. J. Taylor, "Application of computer texture analysis to the singh index," The British Journal of Radiology, vol. 70, pp. 242-247.

[4] S. Sangeetha, J. J. Christopher, and S. Ramakrishnan, "Wavelet based qualitative assessment of femur bone strength using radiographic imaging," International Journal of Biological and Life Sciences, vol. 3 , no. 1 , pp. 276-280, 2007.

[5] M. Anburajan, C. Rethinasabapathi, M. P. Korath, B. G. Ponnappa, A. Govindan, and K. Jagadeesan, "Age related proximal femur bone mineral loss in south indian women: A dual energy X-ray absorptiometry (DXA) study," Journal of Association of Physicians of India, vol. 49, pp. 442-445, 2001.

[6] L. Sooyeul, K. Seunghwan, H. B. Pyo, and S. H. Park, "Bone mineral density estimation from x-ray images," in Proc. the First Joint BMESEMBS Conference Serving Humanity, Advancing Technology, vol. 2, pp. 1040, 1999.

[7] J. T. Pramudito, S. Soegijoko, T. R. Mengko, F. I. Muchtadi, and R. G. Wachjudi, "Trabecular pattern analysis of proximal femur radiographs for osteoporosis detection," Journal of Biomedical and Pharmaceutical Engineering., vol. 1, no. 1, pp. 45-51, 2007. 
[8] T. R. Mengko and J. T. Pramudito, "Implementation of gabor filter to texture analysis of radiographs in the assessment of osteoporosis," MVA2002 IAPR Workshop on Machine Vision Applications, vol. 2, pp. 251-254, 2002

[9] T. R. Mengko and J. T. Pramudito, "Texture analysis of proximal femur radiographs for osteoporosis assessment," WSEAS Transaction on Computers, vol. 3, no. 1, pp. 92-97, 2004.

[10] R. C. Gonzalez and R. E. Woods, Digital Image Processing, 3rd Edition, Prentice Hall, Upper Saddle River, 2008

[11] C. Sinthanayothin, J. F. Boyce, H. L. Cook, and T. H. Williamson, "Automated localization of the optic disc, fovea, and retinal blood vessels from digital color fundus images," Br J Ophthalmol, vol. 83, pp. 902-910, 1999.

[12] C. Chappard, V. Bousson, C. Bergot, D. Mitton, A. Marchadier, T. Moser, C. L. Benhamou, and J. D. Laredo, "Prediction of femoral fracture load: Cross-sectional study of texture analysis and geometric measurements on plain radiographs versus bone mineral density," Radiology, vol. 2, pp. 255-257, 2010.

[13] H. F. Boehm, J. Lutz, M. Korner, W. Mutschler, M. Reiser, and K. J. Pfeifer, "Using radon transform of standard radiographs of the hip to differentiate between post-menopausal women with and without fracture of the proximal femur," Osteoporos Int, vol. 20, pp. 323-333, 2009.

[14] C. Chappard, B. I. Barbara, G. Lemineur, B. Giraudeau, A. Basillasi, R. Harba, and C. L. Benhamou, "Anisotropy changes in post-menopausal osteoporosis: characterization by a new index applied to trabecular bone radiographic images," Osteoporos Int, vol. 16, pp. 1193-1202, 2005.

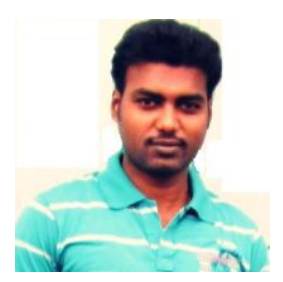

V. Sapthagirivasan is an active researcher, currently pursuing his research in bone densitometry. He did his bachelor's degree in engineering at Madras University and master's degree in engineering at CEG, Anna University 2003 and 2007 respectively. He has over 5 years of experience in research and development of medical image processing. He has more than 15 international conferences in India and other foreign countries and 6 peer reviewed international journals on his credits. His research interests are
Image processing, Computer vision, Pattern recognition, Game theory, Watermarking, Telemedicine, e-Healthcare and informatics.

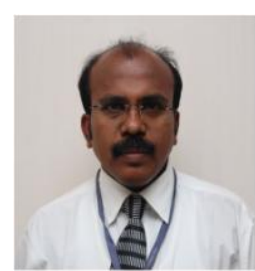

M. Anburajan has obtained his Ph.D. degree in the broad area of 'Medical Physics' from Anna University, Chennai, Tamil Nadu, India in the year 1999. His $\mathrm{PhD}$ thesis entitled as 'Evaluation of Osteoporosis using Conventional $\mathrm{x}$-ray methods and Dual energy x-ray absorptiometry'. He did a Post-Doctoral Research at Division of Medical Physics, Department of Radiology, University Hospital of Kiel, Kiel, Germany in the year 2002. He has completed his M.Sc, and B.Sc Physics from St.Joseph's College, Trichirapalli, Tamil Nadu, in the years 1991, and 1989 respectively. He has 21 years of experience in teaching, research, and hospital experience in Medical Research Institute, Medical College, and Engineering College. He received a Fellow Member of International Medical Science Academy, New Delhi, India. $\mathrm{He}$ is active life member in various societies. His major areas of research are: bone densitometry, medical thermography, and medical imaging devices. He has both published and presented many papers in International/National Journals and Conferences. He was an editor for one monograph and one proceedings of national symposium with ISBN.

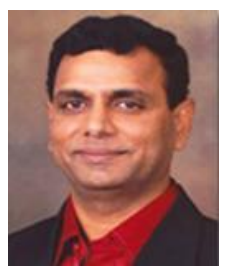

Venkatesh Mahadevan is an experienced academic, and researcher, currently teaching in Australia. He has over 21 years of experience in teaching at higher education and vocational education sector in India, Malaysia, UK, New Zealand and Australia. His research achievements in organizational innovations to date are largely embodied in the strategic planning, alignment, management, execution and quality control assessments of Management Information Systems (MIS). He enjoys collaborating on new innovative multi-disciplinary research themes, which enables him to also put theory into practice to overcome the dilemmas of bridging the gap between the academia and industry. 